\title{
Obstrução nasolacrimal congênita: fatores relacionados com a melhora após sondagem terapêutica
}

\author{
Congenital lacrimal obstruction:outcome and factors associated with \\ therapeuticprobing
}

\author{
Silvana Artioli Schellini ${ }^{1}$ \\ Silvia Narikawa ${ }^{2}$ \\ Sara Capanema Ferreira Ribeiro ${ }^{3}$ \\ Vitor Nakagima ${ }^{4}$ \\ Carlos RobertoPadovani ${ }^{5}$ \\ Carlos Roberto Pereira Padovani ${ }^{6}$
}

\footnotetext{
${ }^{1}$ Professor Livre-Docente do Departamento de Oftalmologia, Otorrinolaringologia e Cirurgia de Cabeça e Pescoço da Faculdade de Medicina de Botucatu da Universidade Estadual Paulista (UNESP). Botucatu (SP).

${ }^{2}$ Residente de Oftalmologia do Departamento de Oftalmologia, Otorrinolaringologia e Cirurgia de Cabeça e Pescoço da Faculdade de Medicina de Botucatu da UNESP. Botucatu (SP).

${ }^{3}$ Residente de Oftalmologia do Departamento de Oftalmologia, Otorrinolaringologia e Cirurgia de Cabeça e Pescoço da Faculdade de Medicina de Botucatu da UNESP. Botucatu (SP).

${ }^{4}$ Professor Assistente do Departamento de Oftalmologia, Otorrinolaringologia e Cirurgia de Cabeça e Pescoço da Faculdade de Medicina de Botucatu da UNESP. Botucatu (SP).

${ }_{5}^{5}$ Professor Titular do Departamento de Bioestatística do Instituto de Biociências da UNESP. Botucatu (SP).

${ }^{6}$ Pós-Graduando do Departamento de Bioestatística do Instituto de Biociências da UNESP. Botucatu (SP).

Endereço para correspondência: Silvana Artioli Schellini. DEP. OFT/ORL/CCP - Faculdade de Medicina de Botucatu - UNESP. Botucatu (SP) CEP 18618-970

E-mail: sartioli@fmb.unesp.br

Recebido para publicação em 22.10.2004

Versão revisada recebida em 08.04.2005

Aprovação em 15.04.2005

Nota Editorial: Depois de concluída a análise do artigo sob sigilo editorial e com a anuência da Dra. Simone H. D. Von Faber Bison e do Dr. Eurípedes da Mota Moura sobre a divulgação de seus nomes como revisores, agradecemos sua participação neste processo.
}

\begin{tabular}{|l|}
\hline RESUMO \\
\hline Objetivo: Avaliar em crianças portadoras de obstrução nasolacrimal con- \\
gênita (ONLC) os índices de cura com a sondagem das vias lacrimais e os \\
fatores relacionados com o insucesso do procedimento. Método: Estudo \\
retrospectivo observacional, incluindo 80 crianças portadoras de obstru- \\
ção nasolacrimal congênita, submetidas à sondagem terapêutica da via \\
lacrimal. As crianças foram avaliadas quanto ao sexo, faixa etária e resul- \\
tado da sondagem. Os dados obtidos foram avaliados por estatística \\
descritiva, teste de Goodman e pelo teste não paramétrico de Mann- \\
Whitney, com nível de significância de $5 \%$. Resultados: A cura ocorreu \\
igualmente em ambos os sexos. A média de idade das crianças que se \\
beneficiaram da sondagem foi de $19,95 \pm 11,4$ meses e a das crianças que não \\
se curaram foi de $23,37 \pm 15,2$ meses. A possibilidade de cura ocorreu \\
igualmente nas faixas etárias acima dos 6 meses. Observou-se nas crianças \\
que não se curaram com a sondagem a existência de alterações nasais como \\
rinite, hipertrofia de adenóide ou decornetos, desvio de septo sinusopatia. \\
Conclusão: A possibilidade de cura com a sondagem não varia significa- \\
tivamente mesmo nas idades acima dos 12 meses. Entre as causas de \\
insucesso com o procedimento devem ser incluídas as alterações da \\
cavidade nasal.
\end{tabular}

Descritores: Obstrução dos ductos lacrimais/congênito; Ducto nasolacrimal; Dacriocistorrinostomia; Fatores de tempo; Falha de tratamento; Estudos retrospectivos

\section{INTRODUÇÃO}

A sondagem lacrimal é o tratamento indicado para a obstrução nasolacrimal congênita (ONLC). Existem duas correntes: uma que defende a sondagem precoce, realizada em recém-natos, no próprio consultório, assim que é detectado o problema, sem anestesia geral ${ }^{(1-2)}$, e outra que acredita ser melhor aguardar até que a criança tenha por volta de um ano de idade para realizar o procedimento. Os defensores da sondagem tardia acreditam que poderá ocorrer cura espontânea na maioria destas crianças, evitando-se procedimentos desnecessários e diminuindo o risco de trauma dos canalículos ${ }^{(3-4)}$.

A literatura sobre o assunto relaciona fortemente os índices de cura obtidos com a sondagem com a idade dos portadores, relatando diminuição da chance de êxito com o aumento da idade. Assim, em crianças com menos de 1 ano é possível haver cura em 95\%; já, nas maiores que 1 ano, apenas $87 \%$ se curam com uma única sondagem ${ }^{(5)}$. Para outros a cura foi de $95 \%$ para menores de 1 ano e $73 \%$ para as maiores de 1 ano $^{(6)}$. Índices de cura de $97 \%$ em crianças sondadas antes dos 13 meses e 54,7\% nas sondadas após 
os 13 meses $^{(7)}$ e de $94 \%$ de cura sondando crianças abaixo de 6 meses, $88,5 \%$ entre 6 meses e 1 ano e 86,5\% em maiores de 12 meses $^{(8)}$ também foram relatados.

Avaliando a resposta ao tratamento da obstrução congênita da via lacrimal excretora foi observado que a chance de cura com a sondagem é semelhante em crianças no primeiro ou no segundo ano de vida ${ }^{(9)}$.

Porém, a prática diária nos tem ensinado que não é apenas a idade o fator que está relacionado com a possibilidade de cura da ONLC, havendo relatos de que o índice de cura não varia significativamente com a idade ${ }^{(10)}$.

Parece que o tamanho do saco lacrimal, assim como o local da via lacrimal onde ocorre a obstrução, também são fatores relacionados com a possibilidade de cura $^{(11)}$.

Portanto, além da idade, devem existir outros fatores que influenciam na possibilidade de cura após a sondagem.

O presente estudo teve por objetivo avaliar o índice de cura, assim como as causas de insucesso com a sondagem terapêutica, em crianças portadoras de ONLC.

\section{MÉTODOS}

Foi feito estudo retrospectivo observacional, incluindo 80 crianças portadoras de ONLC, atendidas no Ambulatório de Vias Lacrimais - Faculdade de Medicina de Botucatu UNESP, no período de 1992 até 2000 e que foram submetidas à sondagem terapêutica das vias lacrimais. As crianças foram avaliadas quanto ao sexo, idade em que foi realizada a sondagem e evolução após o procedimento.

Método utilizado para a sondagem: Criança em decúbito dorsal horizontal e sob anestesia geral inalatória, submetida a cinedacriocistografia usando contraste oleoso (Lipiodol Guerbet Laboratórios Ltda.). Em se constatando a obstrução, realizou-se a dilatação do ponto lacrimal superior ou inferior, introduziu-se a sonda de Bowman 0-00 e efetuou-se a sondagem lacrimal. Imediatamente após a sondagem, a efetividade do procedimento foi investigada com nova cinedacriocistografia.

Avaliação otorrinolaringológica: Quando as crianças não melhoraram, foi feita avaliação nasal por um dos autores (VN), tendo sido realizada rinoscopia anterior.

Análise estatística: Os dados obtidos foram avaliados por meio de estatística descritiva e pelos testes não paramétrico de Mann-Whitney e teste de Goodman. As conclusões foram tiradas no nível de 5\% de significância.

\section{RESULTADOS}

Das 80 crianças analisadas, $42(55,56 \%)$ eram do sexo feminino e 38 (44,44\%), do masculino, sem que houvesse diferença estatística significativa na distribuição entre os sexos. A média de idade dos meninos foi de 20,90 $\pm 11,6$ meses e das meninas, 20,37 $\pm 13,0$ meses ( $p>0,05)$.

A chance de cura com relação ao sexo mostrou que, para ambos os sexos, a possibilidade de cura foi superior a de não cura, sendo de $73,81 \%$ para as meninas e $89,47 \%$ para os meninos, sem que houvesse diferença significativa quanto à possibilidade de cura para ambos os sexos $(p>0,05)$.

Com relação à idade, a média de idade dos que se curaram foi de $19,95 \pm 11,4$ meses e dos que não se curaram foi de 23,37 $\pm 15,2$ meses, com Mediana de 18 e 19 meses, respectivamente. $\mathrm{O}$ valor mínimo para a cura foi de 1 mês e para a não cura de 2 meses de idade e o valor máximo para cura e não cura foi de 48 meses (Tabela 1).

A avaliação comparativa da possibilidade de cura com relação à faixa etária revelou que em 50,0\% das crianças menores que 6 meses, 94,1\% das com idade entre os 6 e 12 meses, $83,3 \%$ das com 12 até 24 meses e $70,6 \%$ das maiores que 24 meses se curaram com o procedimento, sem diferença estatística significativa entre as porcentagens de melhora ou não com a sondagem (resultado estatístico apontado pelas letras minúsculas na tabela 2). Avaliando-se a possibilidade de cura ou de não cura dentro da mesma faixa etária observou-se que para todas as idades acima de 6 meses, a chance de cura com a sondagem predominou sobre a não cura (resultado estatístico apontado pelas letras maiúsculas na tabela 2).

Quando não houve melhora com a sondagem, a pesquisa da causa de insucesso resultou no encontro de alterações nasais, como hipertrofia de cornetos, desvio de septo e sinusopatia, mais freqüentes no sexo feminino (Tabela 3).

\section{DISCUSSÃO}

Apesar da obstrução da drenagem lacrimal em adultos afetar mais as mulheres, a ONLC afeta meninos e meninas na mesma

\begin{tabular}{|lcc|}
\hline \multicolumn{3}{|c|}{ Tabela 1. Medidas descritivas da idade dos portadores de obstrução } \\
nasolacrimal congênita segundo resposta a sondagem terapêutica \\
Medida descritiva & Melhora com sondagem \\
\cline { 2 - 3 } & Sim & Não \\
Valor mínimo & 1 & 2 \\
Mediana & 18 & 19 \\
Valor máximo & 48 & 48 \\
Média e desvio-padrão & $19,95 \pm 11,4$ & $23,37 \pm 15,2$ \\
\hline
\end{tabular}

Tabela 2. Distribuição dos portadores de obstrução nasolacrimal congênita segundo faixa etária e resposta ao procedimento de sondagem

\begin{tabular}{lrccc} 
& \multicolumn{2}{c}{ Melhora com sondagem } & \\
\cline { 2 - 3 } Faixa etária & Sim & Não & Total \\
$<6$ meses & $2(50,0)$ aA & $2(50,0)$ aA & 4 \\
6 meses até 12 meses & $16(94,1)$ aB & $1(5,9)$ aA & 17 \\
13 meses até 24 meses & $35(83,3)$ aB & $7(16,7)$ aA & 42 \\
$>24$ meses & $12(70,6)$ aB & $5(29,4)$ aA & 17 \\
n (\%). & & & \\
Obs.: As letras indicam o resultado do teste estatístico e devem ter a seguinte \\
interpretação: duas letras iguais significam igualdade; duas diferentes refletem \\
diferença estatística. As letras minúsculas comparam os dados nas colunas e \\
as maiúsculas, nas linhas, ou seja, as letras minúsculas comparam o resultado \\
do tratamento nas diferentes faixas etárias e as maiúsculas, a possibilidade de \\
melhora ou não em portadores de mesma idade
\end{tabular}




\begin{tabular}{|c|c|c|}
\hline \multirow[b]{2}{*}{ Alteração } & \multicolumn{2}{|c|}{ Sexo } \\
\hline & Feminino & Masculino \\
\hline Rinite & $1(9,1)$ & $0(0,0)$ \\
\hline Hipertrofia adenóide & $2(18,2)$ & $1(25,0)$ \\
\hline Hipertrofia corneto & $1(9,1)$ & $0(0,0)$ \\
\hline Desvio septo/sinusopatia & $2(18,9)$ & $0(0,0)$ \\
\hline Desvio septo/Hip. adenóide & $1(9,1)$ & $1(25,0)$ \\
\hline Sinusopatia/Hip. adenóide & $0(0,0)$ & $1(25,0)$ \\
\hline Total & $7 / 11(63,6)$ & $3 / 4(75,0)$ \\
\hline Hip.= hipertrofia & & \\
\hline
\end{tabular}

proporção ${ }^{(6)}$, o que pode ser observado em nossa amostra, constituída por ambos os sexos, sem diferença estatística.

A possibilidade de cura com a sondagem também ocorreu igualmente em ambos os sexos, levando à conclusão de que o sexo não é fator determinante para o prognóstico.

As medidas descritivas das crianças avaliadas, mostrou que houve chance igual de cura ou não cura quanto à idade, com relação à idade mínima e máxima. Com relação à média, a cura ocorreu mais em crianças por volta de 19 meses e a não cura, para as crianças que se apresentavam por volta dos 23 meses. Esta constatação está de acordo com a literatura sobre o assunto que aponta índices de cura maiores com idades por volta dos 12 meses e com diminuição destes valores, conforme aumenta a idade da criança $a^{(5,7)}$.

Porém, há evidências de que a idade não é o único fator determinante da possibilidade de cura. Já foi constatado que, sondando crianças menores do que 1 ano, de 1 a 2 anos e maiores do que 2 anos, houve falha estatisticamente semelhante em todos os 3 grupos de crianças $^{(4)}$ e que crianças acima de 1 ano de vida também respondem bem à sondagem ${ }^{(9)}$.

Os resultados obtidos coincidem com outros relatados ${ }^{(4)}$, uma vez que, apesar de observados valores absolutos superiores para crianças entre 6 e 12 meses, o tratamento estatístico mostrou a mesma possibilidade de cura até para crianças acima de 24 meses, fato também comentado por outros ${ }^{(9)}$.

Esta observação nos permite importante conclusão de que se deve tentar a sondagem como procedimento terapêutico até mesmo para crianças com mais que 24 meses de idade, uma vez que existe chance de cura em porcentagem expressiva também nesta faixa etária.

Estudando-se amostra randomizada de crianças portadoras de ONLC, de forma que as que tinham saco lacrimal dilatado foram sondadas imediatamente e as demais, divididas em 2 grupos: sondadas entre 12 e 14 meses e crianças não sondadas e acompanhadas até 24 meses; constatou-se que a resolução espontânea pode ocorrer em crianças de até 24 meses de vida e que não há diferença estatística entre sondar e não sondar com relação à resolução da obstrução ${ }^{(12)}$. Este estu$\mathrm{do}^{(12)}$ e outro ${ }^{(11)}$, realçam o papel do saco lacrimal dilatado como fator que influencia a possibilidade de cura.

Outro fator seria o local da obstrução. Quando a obstrução ocorre no seio de Arlt, a chance de sucesso com a sondagem é de $60 \%$ e quando a obstrução ocorre na válvula de Hasner, a cura ocorre em $100 \%$ das crianças ${ }^{(11)}$. Este fato pode estar relacionado com a presença de problemas mais sérios do que a simples imperfuração da membrana de Hasner, quando o problema ocorre na região proximal ao saco lacrimal.

Outras causas de insucesso com a sondagem seriam as relacionadas com a cavidade nasal. Muitas das crianças aqui estudadas que não se curaram com a sondagem apresentavam alterações nasais, algumas que impossibilitam a drenagem das lágrimas por compressão do óstio, como a hipertrofia dos cornetos ou da adenóide e o desvio de septo. A compressão poderia ser decorrente, também, de processos inflamatórios, como as rinites $\mathrm{e}$ as sinusopatias. $\mathrm{O}$ fato das meninas terem apresentado mais alterações nasais merece melhor investigação.

\section{CONCLUSÃO}

O fator determinante para a possibilidade de cura da ONLC com a sondagem não é apenas a idade da criança, uma vez que se demonstrou haver chance semelhante de resolução antes e depois de 12 meses de vida. Entre as causas de insucesso com o procedimento devem ser incluídas as alterações da cavidade nasal.

\section{ABSTRACT}

Purpose: To determine the success rate of probing for congenital nasolacrimal duct obstruction and the factors related to probe failure. Method: A retrospective observational study was conducted, involving 80 children with congenital nasolacrimal obstruction who underwent nasolacrimal probing. The subjects were evaluated according to sex, age and probing outcome. The data were evaluated according to the descriptive method, Goodman test and Mann-Whitney test adopting a significance of 5\%. Results: The possibility of cure was the same in both sexes. The mean age at cure was $19.95 \pm 11.44$ months and on failure was $23.67 \pm 15.21$ months. The analysis showed no significant change in cure rate when over 6 months old. Nasal alterations such as rinitis, mucosal or turbinate bone hypertrophy, septum deviation and sinusitis were associated with probe failure. Conclusion: The cure rate does not vary significantly at intervals of increasing ages even it over 12 months old. Nasal alterations might be included in the causes of failure.

Keywords: Lacrimal duct obstruction/congenital; Nasolacrimal duct; Dacryocystorhinostomy; Time factors; Treatment failure; Retrospective studies

\section{REFERÊNCIAS}

1. Nucci P, Capoferri C, Alfarano R, Brancato R. Conservative management of congenital nasolacrimal duct obstruction. J Pediatr Ophthalmol Strabismus. 1989;26(1):39-43. 
2. Rehurek J, Holousova M. [Optimization of therapy in lacrimal duct obstruction in neonates and infants]. Cesk Slov Oftalmol 1997;53(6):391-4. Czech.

3. Goldblum TA, Summers CG, Egbert JE, Letson RD. Office probing for congenital nasolacrimal duct obstruction: a study of parental satisfaction. J Pediatr Ophthalmol Strabismus. 1996;33(4):244-7.

4. Zwaan J. Treatment of congenital nasolacrimal duct obstruction before and after the age of 1 year. Ophthalmic Surg Lasers. 1997;28(11):932-6.

5. Robb RM. Success rates of nasolacrimal duct probing at time intervals after 1 year of age. Ophthalmology. 1998;105(7):1307-9; discussion p.1309-10.

6. Mittelman D. Probing and irrigation for congenital nasolacrimal duct obstruction. Arch Ophthalmol. 1996;104(8):1125-6.

7. Katowitz JA, Welsh MG. Timing of initial probing and irrigation in congenital nasolacrimal duct obstruction. Ophthalmology. 1987;94(6):698-705.
8. Stager D, Baker JD, Frey T, Weakley DR Jr., Birch EE. Office probing of congenital nasolacrimal duct obstruction. Ophthalmic Surg. 1992;23(7):482-4.

9. Chaim MP, Bison HDF, Silva JAF. Obstrução congênita do ducto nasolacrimal: estudo de 284 pacientes. Arq Bras Oftalmol. 1996;59(5):519-21.

10. Yap EY, Yip CC. Outcome of late probing for congenital nasolacrimal obstruction in Singapore. Int Ophthalmol. 1997-98;21(6):331-4.

11. Schellini SA, Gaiotto PC, Schellini RC, Moraes-Silva MRB. Obstrução nasolacrimal congênita: diagnóstico e tratamento. Rev Bras Oftalmol. 1994;53(5): 47-53.

12. Young JD, MacEwen CJ, Ogston AS. Congenital nasolacrimal duct obstruction in the second year of life: a multicentre trial of management. Eye. 1996;10(Pt 4): 485-91.

\section{Simpósio Internacional de Córnea e Lentes de Contato - SINCLEC VI Curso Internacional de Glaucoma II Simpósio da Sociedade Ibero-Americana de Glaucoma}

\section{9 e 20 de Maio de 2006}

\section{Instituto de Ensino e Pesquisa do Hospital Sírio Libanês}

São Paulo - SP

IN FO RMAÇÕ ES: JDE Comunicação e Eventos

Tels.: (11) 5084-5284/5084-9174/5082-3030

Fax: (11) 5574-8261

E-mail: jdecomev@uol.com.br 